\section{Case Reports in Ophthalmology}

Case Rep Ophthalmol 2020;11:493-499

\title{
Optical Coherence Tomography Findings (SD-OCT and OCTA) in Early-Stage Type 3 Neovascularization
}

\author{
Nisa Silva Ana Marta Pedro Baptista Maria João Furtado \\ Miguel Lume \\ Serviço de Oftalmologia, Centro Hospitalar e Universitário do Porto, Porto, Portugal
}

\section{Keywords}

Age-related macular degeneration - Early-stage type 3 macular neovascularization - Optical coherence tomography

\begin{abstract}
A 76-year-old male presented with a small hyperreflective density in the outer nuclear layer with subtle retinal pigment epithelium (RPE) elevation and few intraretinal cysts on spectraldomain optical coherence tomography (SD-OCT). Optical coherence tomography angiography (OCTA) confirmed the presence of a tuft-shaped intraretinal neovascular lesion. SD-OCT performed 2 months before showed a smaller RPE elevation at the same location without intraretinal fluid. A 79-year-old male presented with a small hyperreflective density in the outer retina surrounded by scant intraretinal fluid on SD-OCT and a bright vessel on OCTA, suggesting early-stage type 3 neovascularization. SD-OCT performed 2 months before showed a smaller hyperreflectivity at the same location, without intraretinal fluid. An 84-year-old female presented with hyperreflective foci in the outer retina overlying a serous pigment epithelium detachment (PED) with focal RPE disruption on SD-OCT. SD-OCT performed 2 months before showed the same hyperreflective lesion associated with a shallower PED. No neovascular lesions were found on OCTA after six injections of bevacizumab. To conclude, careful evaluation of SD-OCT allows for early detection of type 3 neovascularization at a pre-exudative stage. OCTA may be useful in confirming the presence of intraretinal neovascular lesion and monitoring response to anti-vascular endothelial growth factor agents.




\section{Case Reports in Ophthalmology}

\begin{tabular}{l|l}
\hline DOI: $10.1159 / 000509139$ & (c) 2020 The Author(s). Published by S. Karger AG, Basel \\
\hline
\end{tabular} www.karger.com/cop

Silva et al.: Optical Coherence Tomography Findings (SD-OCT and OCTA) in Early-Stage Type 3 Neovascularization

\section{Introduction}

Advanced age-related macular degeneration (AMD) is one of the leading causes of vision loss in developed countries [1]. Macular neovascularization (MNV) in AMD was initially described and classified by Gass in 1994 [2]. The term retinal angiomatous proliferation was proposed by Yannuzzi and colleagues thereafter [3]. In 2008, Freund et al. [4] observed the presence of retinal neovascular complex without evidence of underlying occult type 1 neovascularization and introduced the term type $3 \mathrm{MNV}$ to define this form of neovascular AMD that involves primarily the neurosensory retina.

According to recent literature, type $3 \mathrm{MNV}$ is a subtype of neovascular lesion occurring in advanced AMD. It seems to originate from deep retinal capillary plexus (DCP), usually in the perifoveal area, in the setting of choroidal and retinal ischemia [5]. Diagnosis of this type of neovascular lesion has been based on findings of dye angiography and spectral-domain optical coherence tomography (SD-OCT) [6]. Detection of small intraretinal vessels in early-stage type $3 \mathrm{MNV}$ using dye-based angiography alone can be challenging. SD-OCT findings, such as cystoid macular edema, intraretinal hyperreflective foci, and pigment epithelium detachment (PED), can aid in the recognition of these lesions. Optical coherence tomography angiography (OCTA) emerged as a promising non-invasive tool to demonstrate the intraretinal neovascular complex in the early phase, often too small to be found with conventional angiography [7].

We aim to report three cases that emphasize the role of SD-OCT in identifying early signs of type 3 neovascularization. Early detection is particularly relevant in this potentially aggressive form of AMD, which commonly affects both eyes over the course of the disease, as prompt treatment can have a positive impact on disease progression and visual prognosis. We also intend to show the importance of OCTA imaging not only in diagnosis but also in monitoring anti-vascular endothelial growth factor agents (anti-VEGF) treatment response in patients with type $3 \mathrm{MNV}$.

\section{Case Reports}

\section{Case Report 1}

A 76-year-old male was diagnosed with neovascular AMD in his left eye (LE) 3 years before. Best-corrected visual acuity (BCVA) was 20/25 in the right eye (RE). SD-OCT of the RE showed a small hyperreflective density in the outer nuclear layer, focal retinal pigment epithelium (RPE) elevation, and focal ellipsoid zone disruption in association with few intraretinal cysts in the overlying neurosensory retina (Fig. 1a, b). OCTA was performed and showed an intraretinal tuft-shaped lesion suggestive of a type $3 \mathrm{MNV}$ lesion (Fig. 1e). The retrospective analysis of SD-OCT performed 2 months before showed a discrete RPE elevation and photoreceptor interruption at the same location, without intraretinal fluid (Fig. 1c). The patient started intravitreal aflibercept treatment on a treat-and-extend regimen in the RE.

\section{Case Report 2}

A 79-year-old male with no history of advanced AMD and no visual complaints presented to routine examination after cataract surgery. Fundus examination of the LE showed a small retinal hemorrhage. BCVA was 20/25 in the LE. SD-OCT revealed a small intraretinal hyperreflective density with focal RPE changes surrounded by scant intraretinal fluid (Fig. 2a, b). The corresponding OCTA scan showed a bright anomalous vessel suggesting early-stage type $3 \mathrm{MNV}$ at the level of the DCP (Fig. 2e). The retrospective analysis of SD-OCT performed 2 


\section{Case Reports in Ophthalmology}

\begin{tabular}{l|l}
\hline DOI: 10.1159/000509139 & (c) 2020 The Author(s). Published by S. Karger AG, Basel \\
\hline
\end{tabular} www.karger.com/cop

Silva et al.: Optical Coherence Tomography Findings (SD-OCT and OCTA) in Early-Stage Type 3 Neovascularization

months before showed a smaller hyperreflectivity in the same location with minimal RPE distortion but without intraretinal exudation (Fig. 2c). Intravitreal treatment with bevacizumab was initiated on a treat-and-extend regimen in the LE.

\section{Case Report 3}

An 84-year-old female with known bilateral non-neovascular AMD developed an exudative lesion in her LE. BCVA was 20/63 in the LE. SD-OCT showed typical findings of type 3 $\mathrm{MNV}$ in the affected eye, including hyperreflective foci in the outer retina overlying a serous PED and RPE disruption (Fig. 3a). The retrospective analysis of SD-OCT performed 2 months before showed the same lesion associated with a shallower PED (Fig. 3b). Intravitreal bevacizumab therapy was initiated on a treat-and-extend regimen in the LE. After the administration of six injections, SD-OCT revealed a significant reduction of the PED height, as well as regression of intraretinal fluid (Fig. 3c). OCTA was also performed at this time of follow-up and no neovascular lesions were found (Fig. 3e, f).

\section{Discussion and Conclusions}

Our study described SD-OCT and OCTA features of early-stage, treatment-naïve type 3 MNV.

Some authors attempted to characterize the early SD-OCT findings of type 3 neovascular lesions. Querques et al. [8] first postulated that a small, localized RPE elevation probably represented the initial lesion on SD-OCT before the development of type $3 \mathrm{MNV}$. Later, Nagiel et al. [9] introduced the term hyperreflective foci to describe punctate hyperreflective density visualized above the external limiting membrane, defined as the earliest sign of nascent intraretinal neovascularization. The hyperreflective foci were thought to consist of intraretinal neovascularization or migrated RPE cells from an underlying PED [9]. As type 3 MNV evolves, focal RPE and photoreceptor disruption occur, followed by cystic changes and thickening of the overlying retina. As they progress, type 3 lesions are usually located between focal RPE defect and the DCP (inner nuclear/outer plexiform layers junction).

OCTA imaging is a valuable tool in the recognition of type 3 neovascularization not only allowing flow detection within type 3 lesions but also defining precise anatomical location. En face OCTA revealed type 3 neovascular lesions originating from DCP [10]. A study by Miere et al. [11] characterized active type $3 \mathrm{MNV}$ as high-flow tuft-shaped vessels originating from DCP, frequently associated with a small clew-like lesion in the choriocapillaris layer. Regarding OCTA features preceding the onset of exudative type $3 \mathrm{MNV}$, Sacconi et al. [12] identified detectable flow on OCTA at the level of hyperreflective foci in nascent type 3 neovascularization followed by progression of the lesion from DCP into the RPE and sub-RPE space (active stage) or rarely regression without functional impairment. The role of OCTA, both en face and transverse scans, has not only been studied in the early recognition of the disease but also in monitoring the response to treatment with anti-VEGF. A work from Miere et al. [13] suggested that neovascular lesions can become undetectable on OCTA after treatment. Recently, Spaide et al. [14] proposed an explanation for these findings considering that vascular remodeling and lesion regression may occur if enough suppression of the neovascular lesion occurs with anti-VEGF treatment. In a study by Han et al. [15], the disappearance of neovascular lesion on OCTA was associated with a lower recurrence rate in comparison to persistent high-flow lesions. Interestingly, Sacconi et al. [16] demonstrated that reappearance of the neovascular complex in recurrent type $3 \mathrm{MNV}$ was possible after the lesion became undetectable in OCTA in response to intravitreal anti-VEGF therapy. The mechanisms involved in the disappearance 


\section{Case Reports in Ophthalmology}

of the neovascular lesion after inhibiting VEGF are not entirely understood. Noteworthy, there are some issues when using OCTA to detect intraretinal neovascularization, such as projection and other artifacts that can led to misinterpretation. Differential diagnosis of this entity from other retinal vascular pathologies is important, in particular from the recently described entity, perifoveal exudative vascular anomalous complex (PEVAC). Type 3 neovascularization may be confused with PEVAC that usually appears as an isolated perifoveal aneurysmal abnormality with flow typically located between superficial and deep retinal plexi on OCTA, typically with no other vascular changes associated. PEVAC features on SD-OCT are distinguishing as it appears as unilateral, round, hyperreflective isolated lesion, often accompanied by intraretinal cystic spaces without any sign of neovascularization or progression into the RPE $[17,18]$.

The findings of our case series are in agreement with previous evidence regarding earlystage type $3 \mathrm{MNV}$. In the three presented cases, retrospective analysis of SD-OCT performed before the development of exudation showed a hyperreflective density located in the outer retina above disrupted RPE, suggesting a nascent type 3 lesion. At the moment of active type $3 \mathrm{MNV}$ diagnosis, two cases underwent OCTA imaging, where a small intraretinal vascular complex emanating from DCP was detected. In the third case, OCTA was only performed after bevacizumab administration. At this stage, after consecutive intravitreal anti-VEGF injections, OCTA showed no evidence of anomalous intraretinal flow suggesting possible regression of the neovascular lesion in response to treatment. This finding must be carefully interpreted, as baseline OCTA is not available and absence of flow in OCTA is different from complete lesion disappearance. Patient monitoring must be pursued in order to detect regrowth and reappearance of neovascularization in OCTA with possible recurrence of exudation.

In conclusion, careful evaluation of SD-OCT allows identification of early signs of type 3 MNV at a pre-exudative stage. The identification of any hyperreflective foci on SD-OCT, in particular in eyes with dry AMD, especially those with thin choroid and subretinal drusenoid deposits, should raise the suspicion of nascent type 3 neovascularization and dictate OCTA imaging. Appropriate follow-up should be carried out and treatment initiated at an early phase of the disease process in order to improve functional results. The effectiveness of anti-VEGF treatment should be monitored with SD-OCT and OCTA imaging.

\section{Statement of Ethics}

This study adhered to the tenets of the 1964 Declaration of Helsinki and was approved by the ethics committee of Centro Hospitalar Universitário do Porto. Written informed consent was obtained from the patients for publication of these case reports. Ethical approval was not required.

\section{Conflict of Interest Statement}

The authors have no conflicts of interest to declare.

\section{Funding Sources}

This research received no specific grant from any funding agency in the public, commercial, or not-for-profit sectors. 


\section{Case Reports in Ophthalmology}

\begin{tabular}{l|l}
\hline DOI: $10.1159 / 000509139$ & $\begin{array}{l}\text { (c) } 2020 \text { The Author(s). Published by S. Karger AG, Basel } \\
\text { www.karger.com/cop }\end{array}$
\end{tabular}

Silva et al.: Optical Coherence Tomography Findings (SD-OCT and OCTA) in Early-Stage Type 3 Neovascularization

\section{Author Contributions}

Nisa Silva: contribution to conception of the work; acquisition, analysis, and interpretation of data; drafting the work. Ana Marta: contribution to acquisition of data; drafting the work. Pedro Baptista: contribution to acquisition of data; drafting the work. Maria João Furtado: contribution to conception of the work; revising critically for important intellectual content. Miguel Lume: contribution to conception of the work; revising critically for important intellectual content; ensuring that questions related to the accuracy or integrity of any part of the work are appropriately investigated and resolved.

\section{References}

1 Bressler NM. Age-related macular degeneration is the leading cause of blindness. JAMA. 2004 Apr;291(15):1900-1.

2 Gass JD. Biomicroscopic and histopathologic considerations regarding the feasibility of surgical excision of subfoveal neovascular membranes. Am J Ophthalmol. 1994 Sep;118(3):285-98.

3 Yannuzzi LA, Negrão S, Iida T, Carvalho C, Rodriguez-Coleman H, Slakter J, et al. Retinal angiomatous proliferation in age-related macular degeneration. Retina. 2001;21(5):416-34.

4 Freund KB, Ho IV, Barbazetto IA, Koizumi H, Laud K, Ferrara D, et al. Type 3 neovascularization: the expanded spectrum of retinal angiomatous proliferation. Retina. 2008 Feb;28(2):201-11.

5 Jackson TL, Danis RP, Goldbaum M, Slakter JS, Shusterman EM, O'Shaughnessy DJ, et al. Retinal vascular abnormalities in neovascular age-related macular degeneration. Retina. 2014 Mar;34(3):568-75.

6 Querques G, Souied EH, Freund KB. How has high-resolution multimodal imaging refined our understanding of the vasogenic process in type 3 neovascularization? Retina. 2015 Apr;35(4):603-13.

7 Kuehlewein L, Dansingani KK, de Carlo TE, Bonini Filho MA, Iafe NA, Lenis TL, et al. Optical coherence tomography aniography of type 3 neovascularization secondary to age-related macular degeneration. Retina. 2015 Nov;35(11):2229-35.

8 Querques G, Querques L, Forte R, Massamba N, Blanco R, Souied EH. Precursors of type 3 neovascularization: a multimodal imaging analysis. Retina. 2013 Jun;33(6):1241-8.

9 Nagiel A, Sarraf D, Sadda SR, Spaide RF, Jung JJ, Bhavsar KV, et al. Type 3 neovascularization: evolution, association with pigment epithelial detachment, and treatment response as revealed by spectral domain optical coherence tomography. Retina. 2015 Apr;35(4):638-47.

10 Tan AC, Dansingani KK, Yannuzzi LA, Sarraf D, Freund KB. Freund KBl. Type 3 neovascularization imaged with cross-sectional and en face optical coherence tomography angiography. Retina. 2017 Feb;37(2):23446.

11 Miere A, Querques G, Semoun 0, El Ameen A, Capuano V, Souied EH. Optical coherence tomography angiography in early type 3 neovascularization. Retina. 2015 Nov;35(11):2236-41.

12 Sacconi R, Sarraf D, Garrity S, Freund KB, Yannuzzi LA, Gal-Or O, et al. Nascent Type 3 Neovascularization in age-related macular degeneration. Ophthalmol Retina. 2018 Nov;2(11):1097-106.

13 Miere A, Querques G, Semoun 0, Amoroso F, Zambrowski O, Chapron T, et al. Optical cohere nce tomography angiography changes in early type 3 neovascularizastion after anti-vascular endothelial growth factor treatment. Retina. 2017 Oct;37(10):1873-9.

14 Spaide RF. New Proposal for the pathophysiology of type 3 neovascularization based on multimodal imaging findings. Retina. 2019 Aug;39(8):1451-64.

15 Han JW, Cho HJ, Kang DH, Jung SH, Park S, Kim JW. Changes in optical coherence tomography angiography and disease activity in type 3 neovascularization after anti-vascular endothelial growth factor treatment. Retina. 2019 May; published ahead of print: https://doi.org/10.1097/IAE.0000000000002562.

16 Sacconi R, Battista M, Borrelli E, Miere A, Corbelli E, Capuano V, et al. OCT-A characterization of recurrent type 3 macular neovascularization. Br J Ophthalmol. 2020;bjophthalmol-2020-316054.

17 Sacconi R, Freund KB, Yannuzzi LA, Dolz-Marco R, Souied E, Capuano V, et al. The Expanded Spectrum of Perifoveal Exudative Vascular Anomalous Complex. Am J Ophthalmol. 2017 Dec;184:137-46.

18 Mrejen S, Le HM, Nghiem-Buffet S, Tabary S, Quentel G, Cohen SY. Insights into perifoveal exudative vascular anomalous complex. Retina. 2020 Jan;40(1):80-6. 


\section{Case Reports in Ophthalmology}
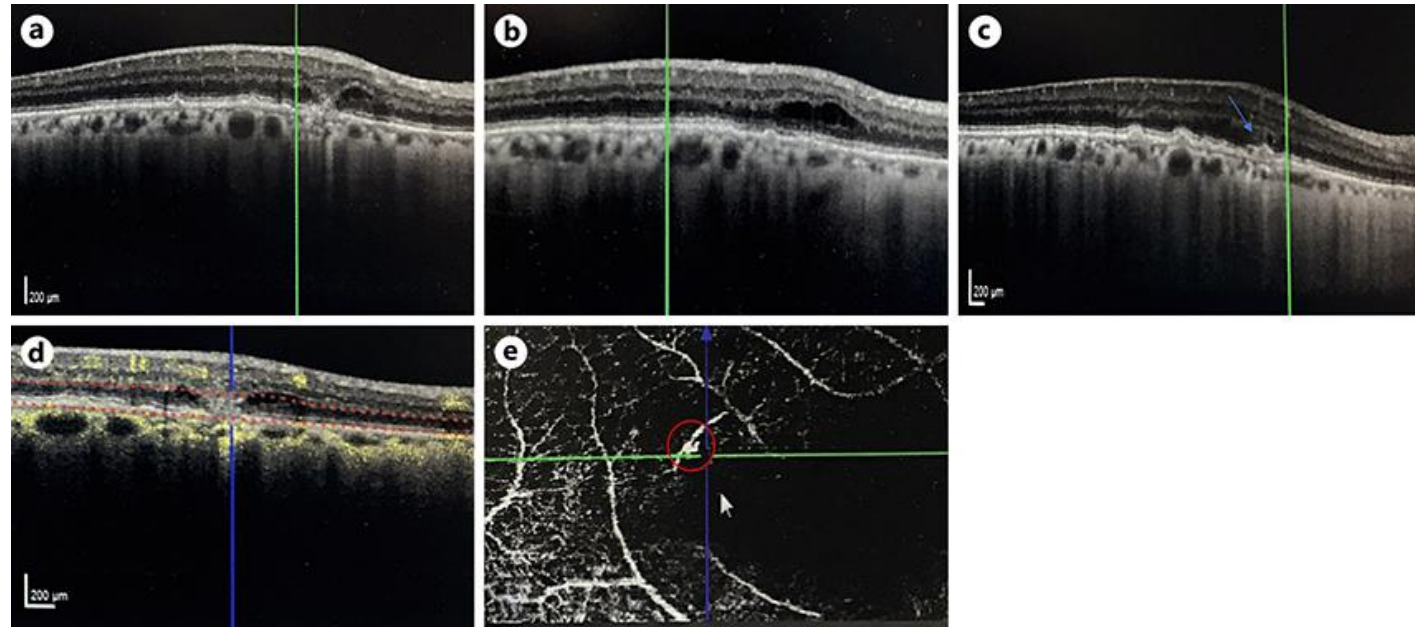

Fig. 1. SD-OCT and OCTA (Heidelberg Engineering, Heidelberg, Germany) imaging of the right eye. a, b SDOCT shows a perifoveal small hyperreflective lesion in the outer nuclear layer with retinal pigment epithelium (RPE) elevation and ellipsoid zone layer discontinuity, as well as thickening and intraretinal cysts of the overlying neurosensory retina. c SD-OCT performed 2 months before shows a discrete RPE elevation in the same location with the focal discontinuity of photoreceptor layers (blue arrow). d OCTA B-scan image. e En face OCTA, after motion and projection artifacts correction, shows a small tuft-shaped vessel in the deep vascular complex slab (red circle).
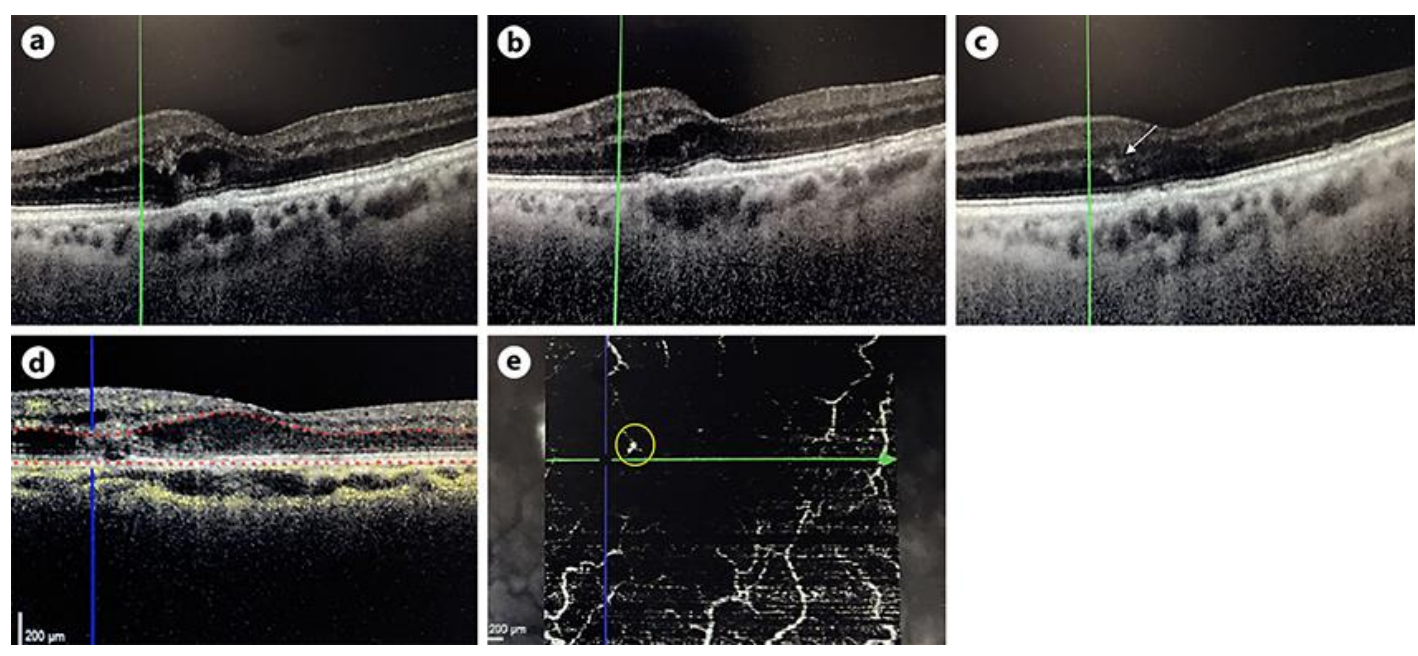

Fig. 2. SD-OCT and OCTA (Heidelberg Engineering, Heidelberg, Germany) imaging of the left eye. a, b SDOCT shows a juxtafoveal hyperreflective lesion in the outer retina surrounded by intraretinal fluid and small drusenoid pigment epithelium detachment. c SD-OCT performed 2 months before shows a smaller hyperreflective density without intraretinal fluid (white arrow). d OCTA B-scan. e En face OCTA, after motion and projection artifacts correction, shows a small bright vessel in the deep vascular complex slab (yellow circle). 


\section{Case Reports in Ophthalmology}
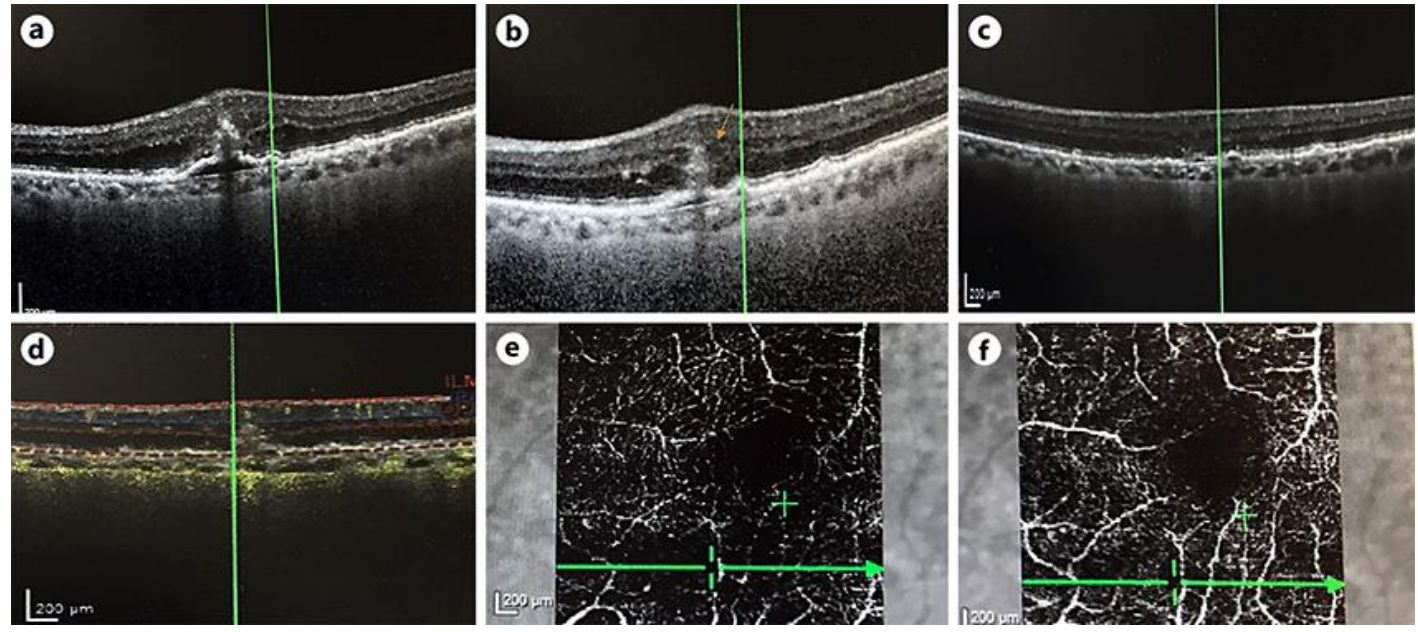

Fig. 3. SD-OCT and OCTA (Heidelberg Engineering, Heidelberg, Germany) imaging of the left eye. a SD-OCT shows a flat serous pigment epithelium detachment (PED) with focal retinal pigment epithelium (RPE) disruption and intraretinal hyperreflective foci overlying the PED with the surrounding fluid. b SD-OCT performed 2 months before shows the same hyperreflective lesion and a shallower PED (orange arrow). c-f SD-OCT and OCTA imaging performed after treatment with bevacizumab. c SD-OCT shows regression of PED, resolution of intraretinal fluid, and a smaller hyperreflectivity also visualized on OCTA B-scan (d); RPE and ellipsoid zone atrophy developed at the level of the previous neovascular lesion. e, f Post-intravitreal treatment en face OCTA after motion and projection artifacts correction. 